\title{
Large-Eddy Simulation of Wake Vortex Evolution from Roll-Up to Vortex Decay
}

\author{
Takashi Misaka*, Frank Holzäpfel ${ }^{\dagger}$ and Thomas Gerz ${ }^{\dagger}$ \\ Deutsches Zentrum für Luft- und Raumfahrt, 82234 Oberpfaffenhofen, Germany \\ Michael Manhart $\ddagger$ \\ Technische Universität München, 80333 München, Germany \\ Florian Schwertfirm ${ }^{\S}$ \\ Kreuzinger+Manhart Turbulenz GmbH, 81675 München, Germany
}

\begin{abstract}
Large-eddy simulation (LES) of wake vortex evolution from vortex generation until decay is performed by combining Reynolds-averaged Navier-Stokes (RANS) simulation and LES. An aircraft model and a surrounding flow field obtained from high-fidelity RANS simulation are swept through a ground fixed computational domain to initialize the wake. After the initialization, time integration of the wake is performed until vortex decay (2-3 minutes). The present paper describes the approach and some results from the simulations where the aircraft is represented by the DLR-F6 wing-body model. The results show that moderate mesh resolution in the wake initialization phase results in lower vortex circulation of wake vortex after roll-up, although the overall distribution of vorticity is not sensitive to the mesh resolution. Boundary condition in the flight direction is one of the key points to continue the time integration of wake vortex after its initialization. Periodic boundary conditions used in this study induce disturbances propagating from boundaries into the computational domain, which leads to faster decay of wake vortex.
\end{abstract}

\section{Introduction}

Wake vortex generated behind a flying aircraft becomes a potential risk for the following aircraft. ${ }^{1}$ In addition, it is pointed out that condensation trails (contrails) originated from aircraft may trigger the formation of cirrus clouds (contrail cirrus), which have been suspected to have influence on climate. ${ }^{2}$ Wake vortex is related to a broad scale of flows ranging from few millimeters to several tens kilometers. Specifically, flows around aircraft's main wing, fuselage, slat, flap, jet engine and tail plane, and their interactions may affect the generation of wake vortex in particular in a high-lift condition. ${ }^{3}$ On the other hand, contrails generated by cruising aircraft spread several tens kilometers. ${ }^{2}$

The formation process of wake vortex is divided into four regimes: ${ }^{4}(1)$ jet regime, (2) vortex regime, (3) dissipation regime, and (4) diffusion regime. Although numerical simulation of the atmosphere is one of the effective approaches to tackle this problem, the applicable flow scale of a numerical simulation code is usually limited to each of those regimes. High-fidelity Reynolds-averaged Navier-Stokes (RANS) simulations could handle flows around aircraft and subsequent roll-up process of wake vortex in the jet regime. Then, the time evolution of wake vortex is continued by large-eddy simulation (LES). ${ }^{5}$ On the other hand, dynamics of wake vortex in the vortex and dissipation regime has been studied mainly by LES or direct numerical simulation (DNS). In these researches, detailed time evolution of a vortex pair with a longitudinally constant velocity profile is investigated, where short-wave (elliptic) instability ${ }^{6-8}$ and Crow instability ${ }^{9,10}$ may develop. In

\footnotetext{
*Research Assistant, Institut für Physik der Atmosphäre, Münchner Straße 20, Member AIAA.

$\dagger$ Research Scientist, Institut für Physik der Atmosphäre, Münchner Straße 20, Member AIAA.

${ }^{\ddagger}$ Professor, Fachgebiet Hydromechanik, Arcisstraße 21.

$\S$ Consulting Engineer, Kirchenstraße 34 .
} 
addition, various atmospheric conditions of turbulence, stability and wind shear are considered to assess the effect of these factors on wake vortex evolution and decay. ${ }^{11-13}$ Large-eddy simulation of wake vortex in the late dissipation and diffusion regimes is performed along with microphysical processes of contrails, where meteorological features of a numerical simulation code are important. ${ }^{14}$

This study describes a consistent approach to simulate wake vortex from its generation until vortex decay which is under development. The approach is based on a combination of high-fidelity RANS simulation which can produce a detailed flow field around aircraft and LES which can realize longer vortex age until vortex decay (2-3 minutes). The integration of RANS and LES is conducted by using the RANS solution as a forcing term in the LES computation. Similar approach might be referred to as the fortified solution algorithm (FSA), ${ }^{15}$ or a nudging technique used in meteorological community. ${ }^{16}$ To initialize aircraft's wake, the current approach employs a ground fixed computational domain and the RANS solution is swept through the domain as if it would be flying through. Then, LES computation is continued to simulate wake vortex until its decay. The ground fixed LES domain makes possible to use experiences on the previous wake vortex researches such as an ambient turbulence generation methodology, vortex tracking and evaluation methods. ${ }^{17}$ This paper describes the approach and some results from simulations using a RANS solution around the DLR-F6 wing-body model.

\section{Methods}

\section{II.A. Governing equations and numerical methods}

LES is performed by using incompressible Navier-Stokes code MGLET developed at Technische Universtät München. ${ }^{18}$ To perform wake vortex simulation in various atmospheric conditions, an equation for potential temperature is also solved to take into account buoyancy effects (Boussinesq approximation).

$$
\begin{gathered}
\frac{\partial u_{i}}{\partial t}+\frac{\partial\left(u_{i} u_{j}\right)}{\partial x_{j}}=-\frac{1}{\rho_{0}} \frac{\partial p^{\prime}}{\partial x_{i}}+\nu \frac{\partial^{2} u_{i}}{\partial x_{j}^{2}}+g \frac{\theta^{\prime}}{\theta_{0}} \delta_{i 3}, \\
\frac{\partial \theta^{\prime}}{\partial t}+\frac{\partial\left(u_{j} \theta^{\prime}\right)}{\partial x_{j}}=\kappa \frac{\partial^{2} \theta^{\prime}}{\partial x_{j}^{2}}+u_{3} \frac{d \theta_{s}}{d x_{3}}, \\
\frac{\partial u_{j}}{\partial x_{j}}=0,
\end{gathered}
$$

where $u_{i}, p^{\prime}$ and $\theta^{\prime}$ represent velocity components in three spatial directions ( $i=1,2$ or 3 ), pressure and potential temperature, respectively. Summation convention is used for velocity components $u_{i}$ and $\delta_{i j}$ denotes Kronecker's delta. The primes for pressure and potential temperature show that these are defined by the deviation from the reference states: $p=p_{0}+p^{\prime}, \theta=\theta_{0}+\theta^{\prime}$. In this study, typical values of air density and potential temperature at flight altitude are employed; $\rho_{0}=0.35 \mathrm{~kg} / \mathrm{m}^{3}, \theta_{0}=332.1 \mathrm{~K} .{ }^{19} \mathrm{In}$ the Boussinesq approximation, the potential temperature is coupled to momentum equations through the vertical velocity component. The resulting equation set is energy conserving system. ${ }^{20}$ Kinematic viscosity in Eq. (1) is defined by the sum of molecular viscosity and eddy viscosity obtained by a subgrid-scale model. Corresponding diffusion coefficient $\kappa$ in Eq. (2) is obtained by assuming constant molecular and turbulent Prandtl numbers of 0.7 and 0.9 , respectively. In addition to the above, equations for passive tracer are employed in which velocity induced advection and diffusion by molecular and turbulent viscosity are considered.

The above equations are solved by a finite-volume approach with the fourth-order finite-volume compact scheme. ${ }^{21,22}$ A split-interface algorithm is used for the parallelization of the tri-diagonal system, which realize smaller overhead time and scalability in parallel environment compared to the existing parallel tri-diagonal matrix solvers. ${ }^{23}$ In addition, a divergence free interpolation is employed for obtaining advection velocity, which ensures conservation of velocity and passive tracer fields. A pressure field is obtained by the velocitypressure iteration method by Hirt et al. ${ }^{24}$ The iteration is performed until the divergence of the velocity field becomes smaller than a threshold value of $1.0 \times 10^{-5} \mathrm{~s}^{-1}$. The third-order Runge-Kutta method is used for the time integration. ${ }^{25}$ Lagrangian dynamic model is employed for a turbulence closure. ${ }^{26}$ The use of standard Smagorinsky model results in excessive eddy viscosity in the center of vortex, hence, a correction procedure is usually used together with the standard Smagorinsky model. ${ }^{27,28}$ An alternative way to handle vortex flow is the use of a dynamic-type subgrid scale model as in the present study. The Lagrangian dynamic model does 
not require specific direction for the averaging process of subgrid model coefficients which is usually required in dynamic-type models for stable computation, therefore, the Lagrangian dynamic model is appropriate for wake vortex simulation where there is no relevant direction for the averaging. All computations are performed in parallel by a domain decomposition approach.

\section{II.B. Integration of RANS and LES solutions}

In this study, a RANS solution of the DLR-F6 wing-body model is employed to initialize its wake in a computational domain. The RANS solution is obtained by the DLR TAU-code with hybrid unstructured mesh, where the number of mesh points is approximately 8.5 million. ${ }^{29}$ The flow conditions of Mach number $M=0.75$ and Reynolds number $\mathrm{Re}=5.0 \times 10^{6}$ are considered here. Vorticity distribution from the RANS solution is shown in Fig. 1. Since LES of wake vortex is performed in dimensional scale, the model and the surrounding flow field are simply scaled so that the span becomes realistic value, that is, $60.3 \mathrm{~m}$ is assumed in this study.

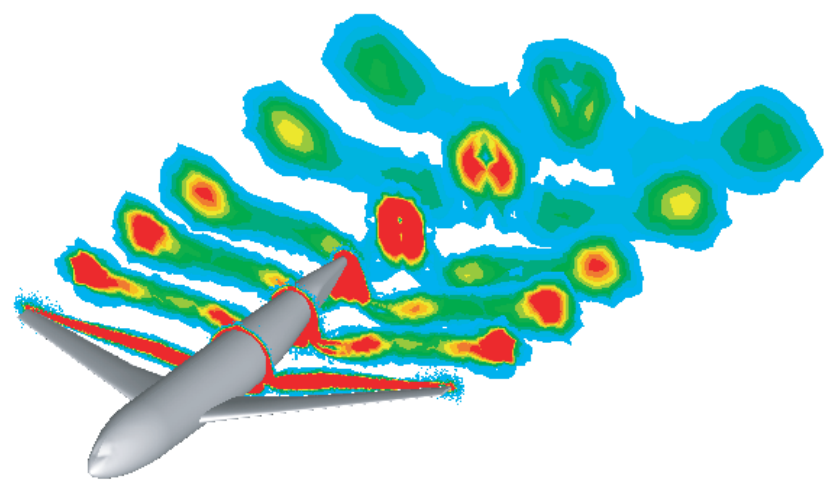

Figure 1. Vorticity distribution from RANS solution of the DLR-F6 model.

The flow field obtained from RANS simulation is integrated to LES by replacing the RANS solution with LES solution in the region close to wall surface of the model. It is simply expressed as follows:

$$
\boldsymbol{V}=f(y) \boldsymbol{V}_{\mathrm{LES}}+[1-f(y)] \boldsymbol{V}_{\mathrm{RANS}} .
$$

Here, a velocity field is represented by a combination of RANS velocity field $\boldsymbol{V}_{\text {RANS }}$ and LES velocity field $\boldsymbol{V}_{\text {LES }}$ with a weighting function $f(y)$. Figure 2 shows a schematic of the weighting function around an object. In this study, $\boldsymbol{V}_{\text {RANS }}$ is provided as a constant forcing term of Navier-Stokes equations solved in the LES. Since the aircraft model is swept through a computational domain, the forcing term of a RANS solution acts as a moving boundary condition for the LES. This kind of approach might be referred as the fortified solution algorithm, ${ }^{15}$ or a nudging technique in meteorological community. ${ }^{16}$ And a combination of RANS and LES through a boundary condition ${ }^{30}$ would be referred as an approach similar to the present study although its details are different. We do not take the approach of hybrid LES-RANS method for turbulent flows because of limited mesh resolution in the present LES, that is, a boundary layer profile around the model is not handled by the LES mesh. In addition, only a one-way coupling of RANS and LES solutions is used.

The threshold for switching the solutions could simply be a distance from body surface, or other physical quantity such as velocity magnitude. Here, we employed the following function to enable a smooth transition between the solutions.

$$
f(y)=\frac{1}{2}\left[\tanh \left[A\left(\frac{y}{d_{w}}-\frac{d_{w}}{y}\right)\right]+1.0\right],
$$

where a hyperbolic tangent function is used to realize smooth transition from RANS to LES. The constants $A$ and $d_{w}$ represent slope of the transition and wall-distance to switch the solutions, respectively. Figure 3 shows iso-surfaces of wall-distance $d_{w}=4.0 \mathrm{~m}$, where solutions of RANS and LES are equality weighted. Currently, relatively large $d_{w}$ is employed because mesh resolution in the LES is limited to the order of $1.0 \mathrm{~m}$ and larger overlap region is required to enforce RANS solution in LES computation. 


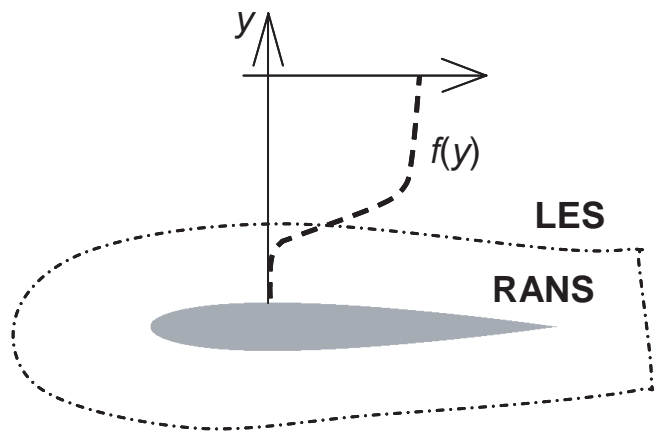

Figure 2. Schematic of a weighting function and a combination of RANS and LES solutions.

It should also be mentioned that the current approach uses a combination of compressible and incompressible codes, which is because of the requirement of large time step in wake vortex simulation. In a ground fixed frame, a flow around an aircraft model is dragged with the speed of flight (here, $270 \mathrm{~m} / \mathrm{s}$ ), and the influence is reduced as the distance from the model increases. Therefore, the threshold $d_{w}$ has a role that the region with low flow velocity is solved by an incompressible code in the LES part. This is one of the reasons to choose a ground fixed frame for wake initialization. The CFL condition is applied to the moving aircraft model. The advancements of 1.0 and $0.5 \mathrm{~m}$ mesh width per time step do not show large difference in the initialized wake.

For using the unstructured RANS solution from TAU-code in the Cartesian mesh LES, a mapping of flow quantities of the unstructured mesh onto the Cartesian mesh is performed. It is done by a linear interpolation only once before starting a new simulation (new RANS solution). Figure 4 shows the DLR-F6 model geometry and examples of Cartesian meshes with a mesh spacing of 0.5 and $1.0 \mathrm{~m}$. Even with $0.5 \mathrm{~m}$ mesh spacing, the mesh resolution is coarse compared to DLR-F6 model geometry and unstructured mesh around it (the unstructured mesh is not shown here). The mesh resolution in the order of $1.0 \mathrm{~m}$ is a typical value for the LES initialized with a fully rolled-up wake pair. To fill the gap of mesh resolution, zonal mesh refinement or mesh stretching would be required.

(a) Perspective view

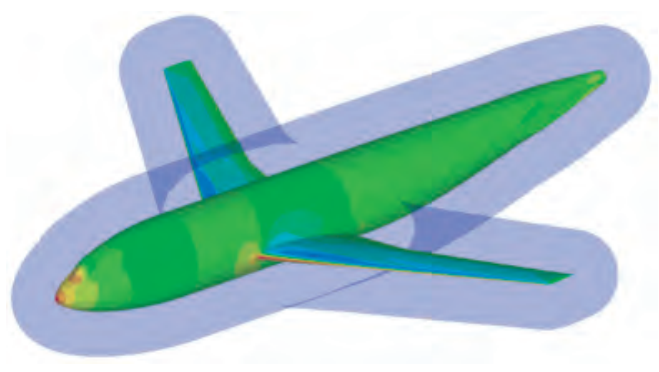

(b) Top view

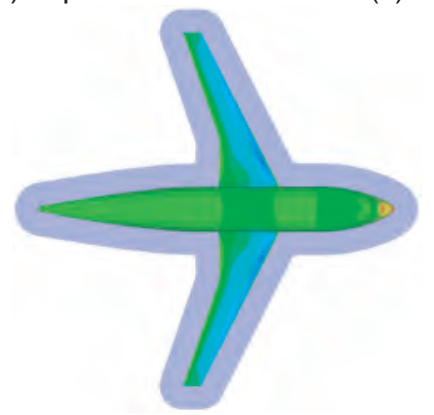

(c) Front view

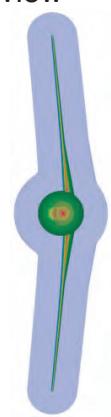

Figure 3. Distribution of the threshold $d_{w}=4.0 \mathrm{~m}$ used for Eq. (5).

The procedure of the simulation can be divided into two phases. In the first phase, an aircraft model starts to move impulsively from the one side of a computational domain. After the movement of the domain length, the aircraft model comes back to its original position because of periodic boundary conditions as shown in Fig. 5(a). The solution integration procedure described above is applied in this phase to initialize the wake of an aircraft model. In the second phase, a right side part of the domain (within red dashed line) in Fig. 5(a) is cut out to produce a smaller domain which does not include the aircraft model used for wake initialization. Then, LES is continued in the small domain to simulate wake vortex evolution. Since the interaction of an aircraft model and its own wake occurs as shown in Fig. 5(a), relatively large domain length 
(a) $1.0 \mathrm{~m}$ mesh

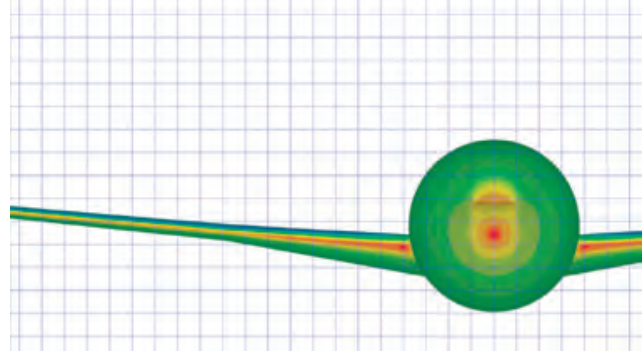

(b) $0.5 \mathrm{~m}$ mesh

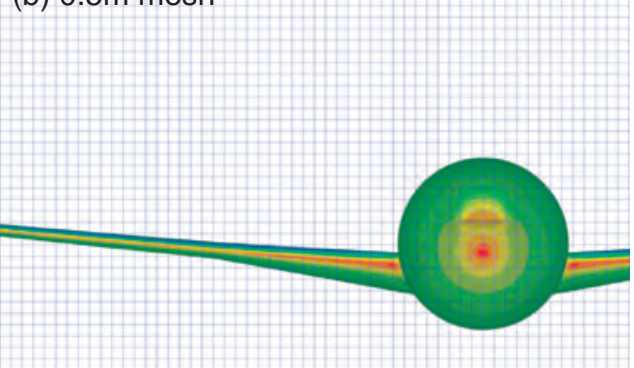

Figure 4. DLR-F6 model geometry and Cartesian meshes for a mapping of RANS solution.

should be used during the wake initialization phase to avoid disturbances in the small domain. Appropriate boundary condition for the small domain is one of the crucial points in the second phase. Currently, we have used periodic boundary condition because of its stability in wake vortex flows. Since there is a certain time-lag between left and right boundaries in Fig. 5(b), the application of periodic boundary condition is not favorable. On the other hand, open boundary condition based on the extrapolation of flow variables could be used, however, the stable computation of wake vortex which directly connected to the boundaries is not straight forward. The influence of periodic boundary condition in the second phase is discussed later.

The dimensions of the computational domain in Fig. 5(a) are 576, 192 and $192 \mathrm{~m}$ in the flight, span-wise and vertical directions, respectively. And the lengths in Fig. 5(b) are 400, 192 and $192 \mathrm{~m}$ in the three directions. A uniform mesh spacing of $1.0 \mathrm{~m}$ in all three directions is employed unless otherwise stated. The mesh resolution of $1.0 \mathrm{~m}$ is reasonable for wake vortex simulations in the vortex regime, however, it might not be enough for the simulation of the jet phase where the roll-up occurs. The domain length in the flight direction in Fig. 5(b) covers a theoretical wave length of the Crow instability. ${ }^{31}$

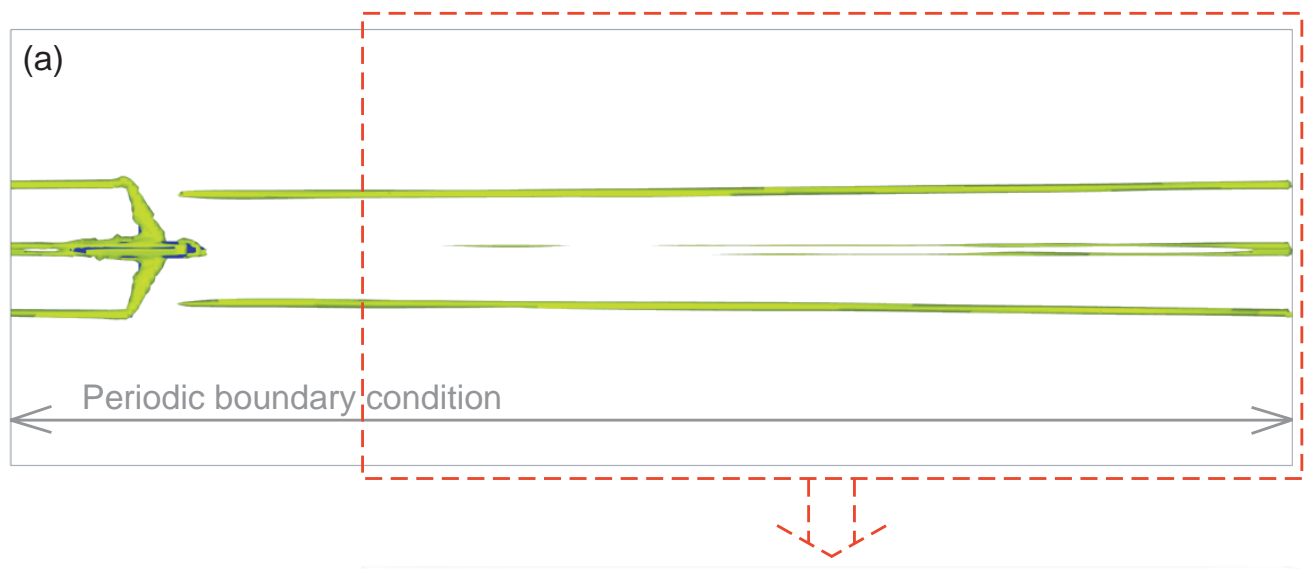

(b)

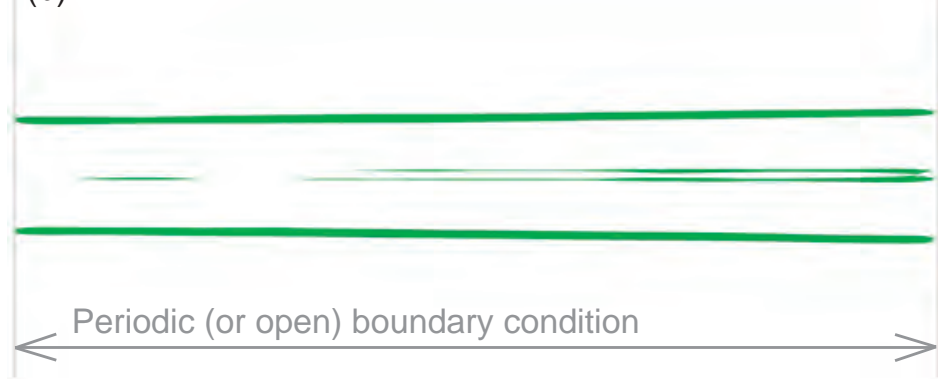

Figure 5. Schematic for switching procedure from wake initialization to wake vortex evolution phase. 


\section{Results}

\section{III.A. Roll-up process of wake vortex}

Figure 6 shows vorticity magnitude within planes at three different positions where the DLR-F6 model flies through in time. The interval of the planes is $40 \mathrm{~m}$. Large vorticity produced from wing-tip and fuselage appears in the planes after the passage of the DLR-F6 model. Note that the number of mesh points is halved in each spatial direction in Fig. 6. It is confirmed that the fuselage produces relatively large vorticity in the wake. The resolution of the Cartesian mesh affects the resolution of wake, therefore, the wake of fuselage might become tighter with finer Cartesian mesh. In addition, relatively large vorticity is produced behind inboard wing.

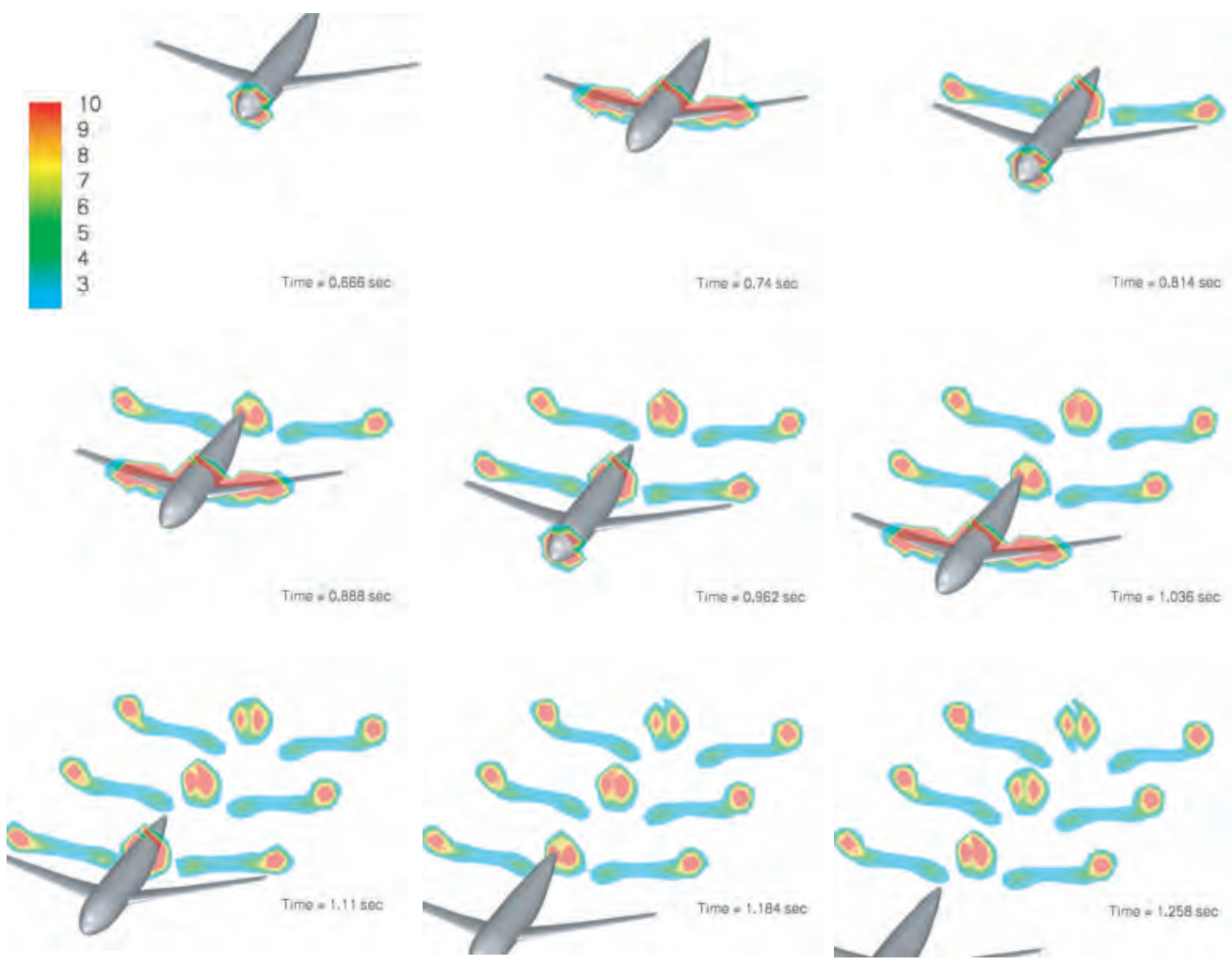

Figure 6. Vorticity magnitude within three planes where the DLR-F6 model flies through. Note that the number of mesh points is halved in each spatial direction.

Figure 7 shows the vorticity distributions on several downstream planes which are obtained from the computations with different mesh resolutions of 0.5 and $1.0 \mathrm{~m}$. Here, the origin of the coordinate in the flight direction is set to trailing edge of wing-tip and the downstream distance $x$ is normalized by wingspan $b$. Boundary layer around fuselage appears as high vorticity magnitude in both mesh resolutions. The boundary layer of $0.5 \mathrm{~m}$ mesh case shows finer distribution compared to that of $1.0 \mathrm{~m}$ at $x / b=0.0$, however, the thickness of boundary layer is still large in comparison with the RANS solution shown in Fig. 1. It is due to less resolution of Cartesian mesh. At $x / b=1.0$, peaks of vorticity magnitude from wing-tip and fuselage are kept sharp in the $0.5 \mathrm{~m}$ mesh case. Roll-up of wing-tip vortex proceeds as shown in $x / b=3.0$. Overall vorticity distribution is similar in both cases, however, less captured vorticity peak might affect time evolution of wake vortex at a later time.

Figure 8 shows the influence of parameters in Eq. (5) on the vorticity distribution downstream $x / b=1.0$. As shown in the figures, a larger threshold value $d_{w}$ and smoother transition of a tangent hyperbolic function realize smoother vorticity distribution in the downstream plane. In $d_{w}=4.0 \mathrm{~m}$ and $A=2.0$ case shown in Fig. 8(b), vorticity disturbance appears above the wake of the main wing. This is because a high speed flow over the main wing is solved by a incompressible code. On the other hand, such kind of vorticity disturbance is not seen in $d_{w}=6.0 \mathrm{~m}$ and $A=1.0$ case as shown in Fig. $8(\mathrm{c})$. 

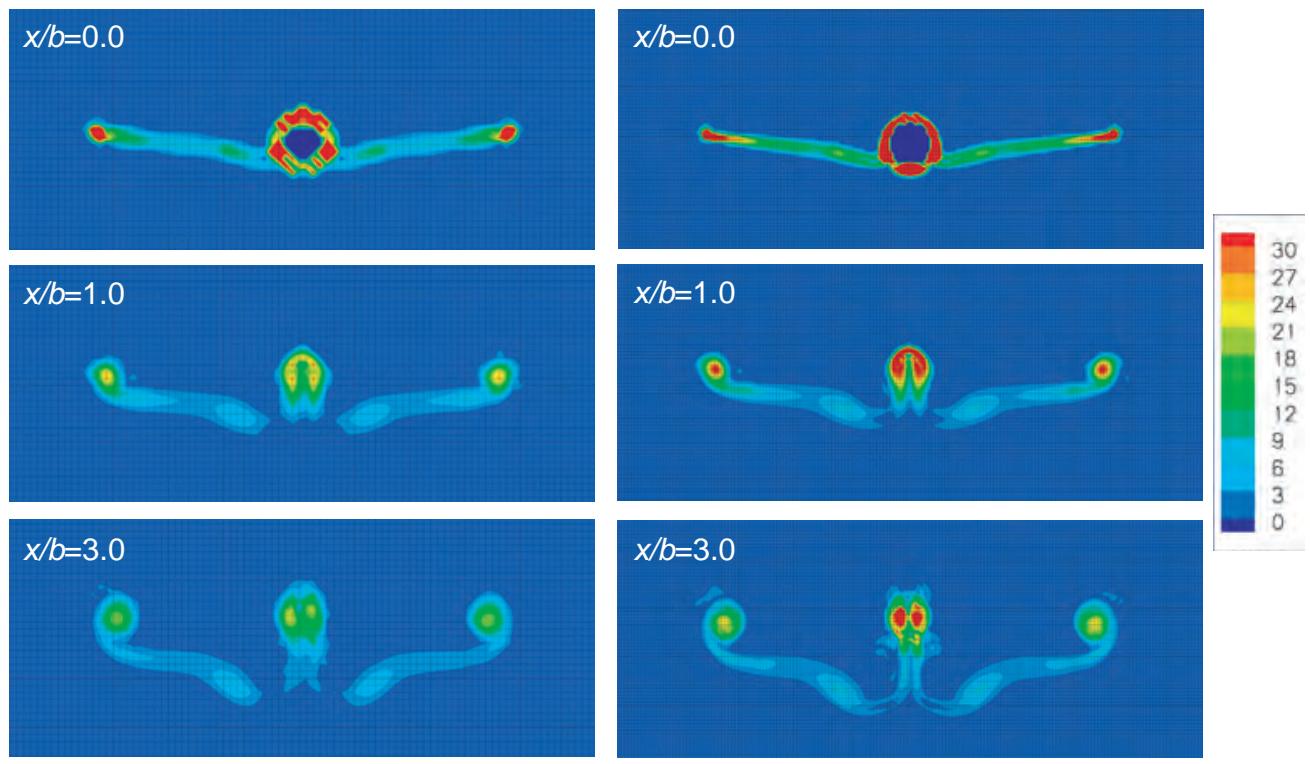

(a) $1.0 \mathrm{~m}$ mesh

(b) $0.5 \mathrm{~m}$ mesh

Figure 7. Vorticity distribution on several downstream planes with two different mesh resolutions of 0.5 and $1.0 \mathrm{~m}$.
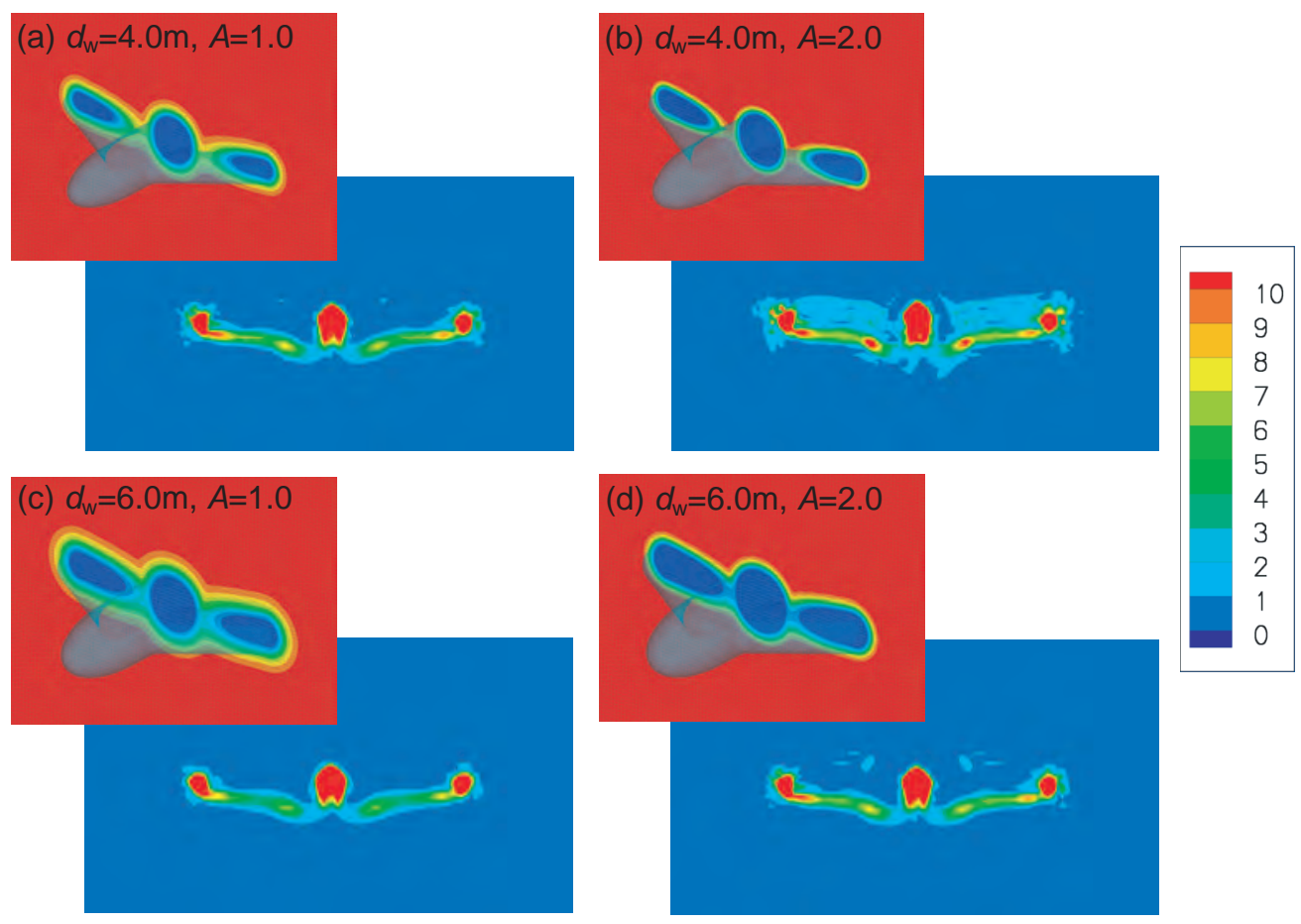

Figure 8. The influence of RANS-LES transition parameters in Eq. (5) on the vorticity distribution in downstream planes. 


\section{III.B. Time evolution until vortex decay}

Figure 9 shows the time evolution of vorticity distribution on a ground fixed vertical plane from $T=2.1$ until $38.9 \mathrm{~s}$, which correspond to the distances of 567 to $10,503 \mathrm{~m}$ from the aircraft model, respectively. Vorticity from fuselage decays quickly as shown in Fig. 9(b), on the other hand, the vorticity from wing-tips is preserving its peak value. The vorticity from inboard wing rotates around wing-tip vortex because of strong circulation induced by the wing-tip vortex. In the later time, the flow field is reorganized to single vortex pair, which is often assumed in the simulation study of wake vortex.

(a) $T=2.11 \mathrm{sec}$

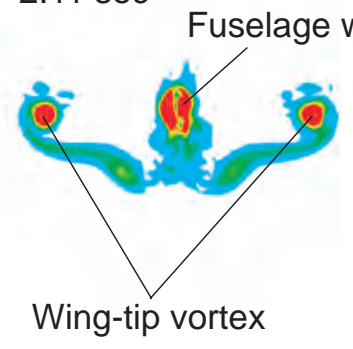

(d) $T=19.35 \mathrm{sec}$

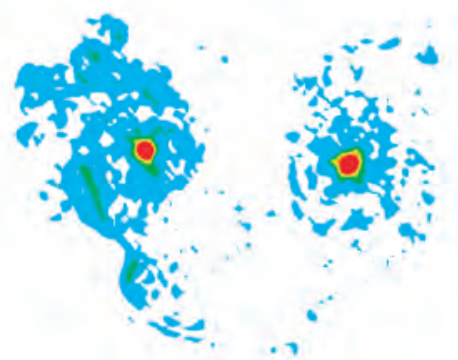

(b) $T=4.35 \mathrm{sec}$

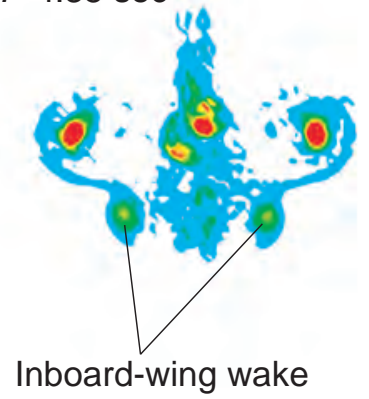

(e) $T=28.35 \mathrm{sec}$ (c) $T=9.60 \mathrm{sec}$

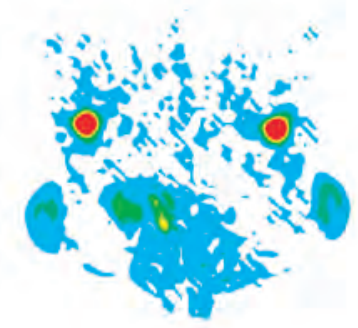

(f) $T=38.85 \mathrm{sec}$
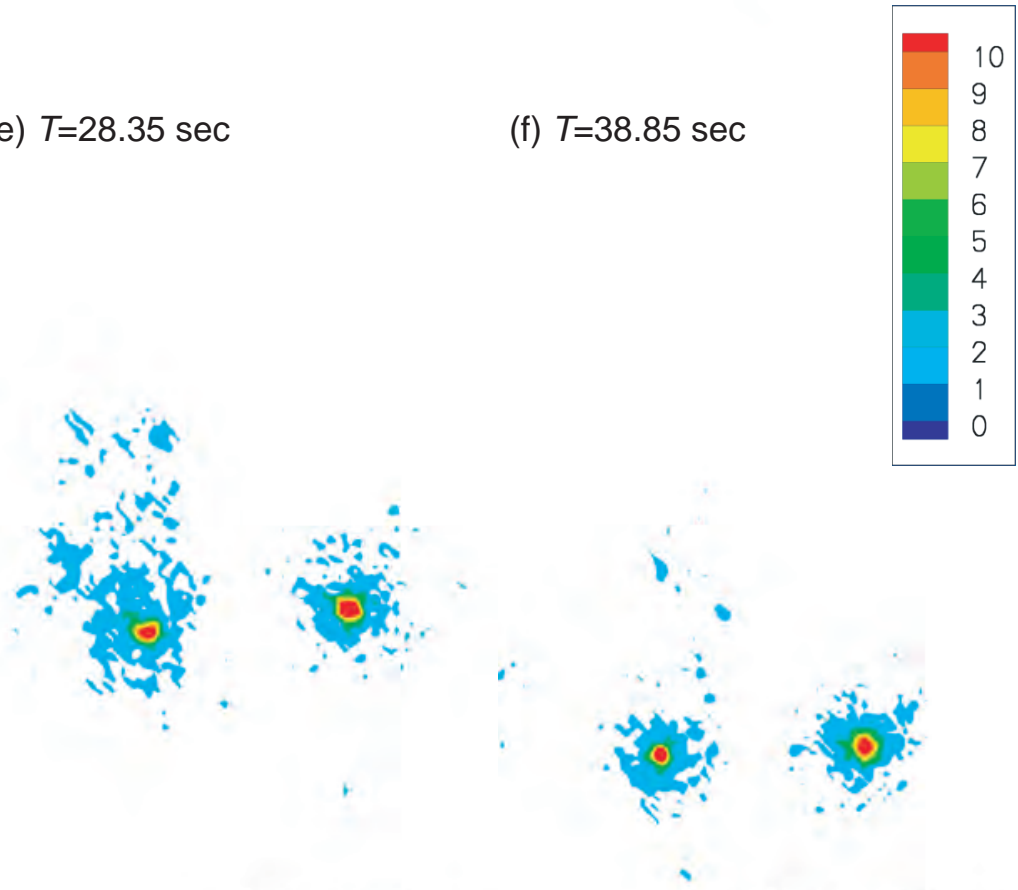

Figure 9. Time evolution of vorticity magnitude distribution on a ground fixed vertical plane.

Figure 10 shows the time evolution of wake vortex by iso-surfaces of vorticity magnitude where positive and negative values from each vorticity component are displayed. These figures show a view from the top. It is confirmed from Fig. 10(a) that the wake develops during a flight of $400 \mathrm{~m}$, i.e., only $1.48 \mathrm{~s}$ in the present case. As shown in Fig. 10(b), the wake of fuselage is quickly disturbed. At the same time, wing-tip vortices are also disturbed near boundary, which is due to the application of periodic boundary condition. The development of wake during the flight violates the periodicity of the flow. For the sake of reasonably stable computation, we employed periodic boundary condition in this study. The disturbed fuselage wake and vorticity from an inboard wing rotate around wing-tip vortex as shown in Figs. 10(c) and (d). In Fig. 10(e), a single vortex pair is reorganized and the deformation starts to appear. There are secondary vortices 
wrapping around wing-tip vortex, which is known as a mechanism of wake vortex decay in a strong ambient turbulence condition. The secondary vortices appear from disturbances at the boundaries and partially from highly disturbed fuselage wake. Finally, the vortex pair is largely deformed and the vorticity strength is weakened.

Figure 11 shows the time evolution of vortex parameters, averaged circulation $\Gamma_{5-15}$ and vortex core radius. The value of circulation is smaller than the value of $548 \mathrm{~m}^{2} / \mathrm{s}$ estimated from the lift coefficient $C_{L}=0.5$ assuming an elliptic load distribution. It is due to the lack of mesh resolution during the wake initialization phase and a subsequent loss of vorticity from wing-tip vortex. The evolution of vortex core radius is reasonable compared to the previous experimental and numerical researches, however, the core radius might appear smaller with the use of finer mesh.

(a)

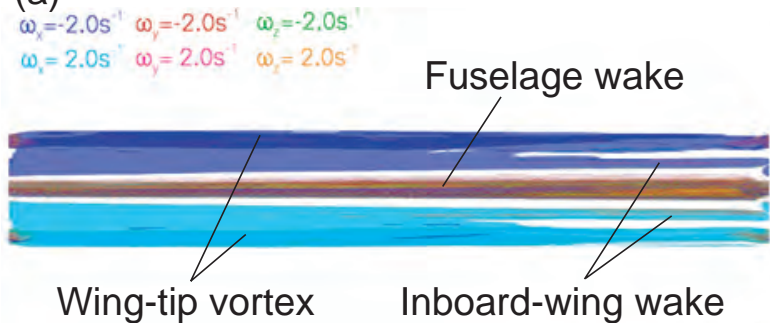

(c)

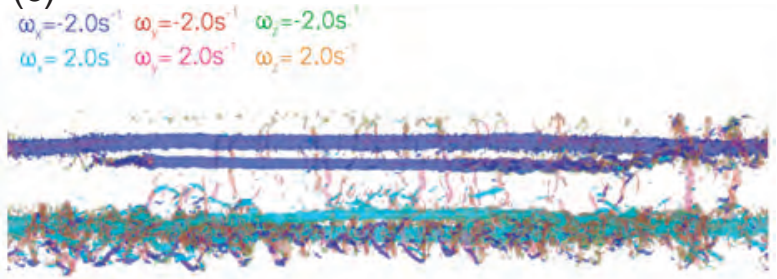

(e)

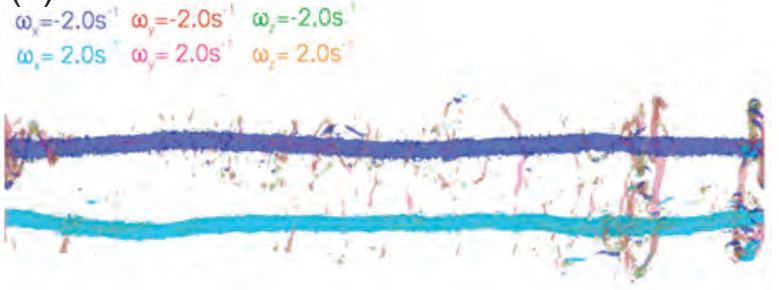

(g)

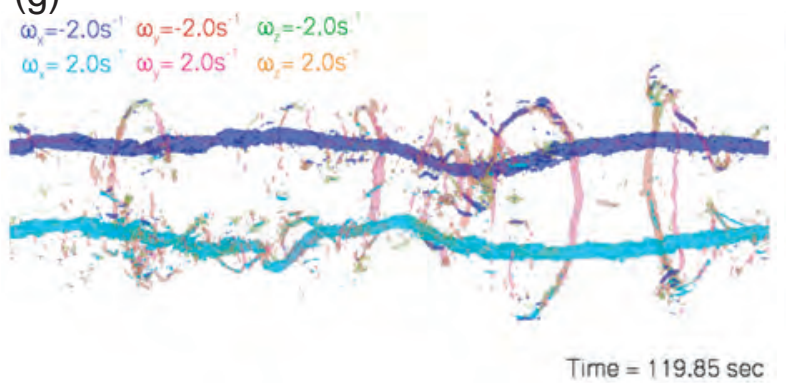

(b)

$\omega_{x}=-2.0 s^{-1} \quad \omega_{y}=-2.0 s^{-1} \quad \omega_{z}=-2.0 s$

$\omega_{1}=2.0 \mathrm{~s}^{\mathrm{s}} \quad \omega_{\mathrm{y}}=2.0 \mathrm{~s}^{-1} \quad \omega_{2}=2.0 \mathrm{~s}$

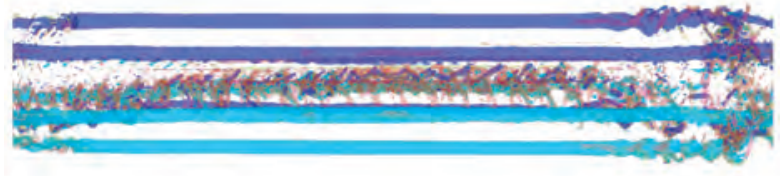

(d)

$\omega_{x}=-2.0 \mathrm{~s}^{-1} \quad \omega_{y}=-2.0 \mathrm{~s}^{-1} \quad \omega_{z}=-2.0 \mathrm{~s}$.

$\omega_{2}=2.0 \mathrm{~s}^{\prime} \quad \omega_{y}=2.0 \mathrm{~s}^{-1} \quad \omega_{2}=2.0 \mathrm{~s}^{-1}$

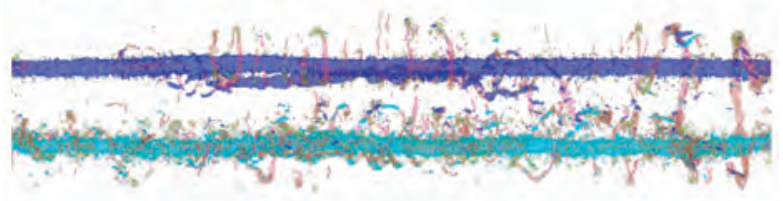

(f)

$\omega_{y}=-2.0 \mathrm{~s}^{-1} \omega_{\mathrm{y}}=-2.0 \mathrm{~s}^{-1} \quad \omega_{2}=-2.0 \mathrm{~s}$

$\omega_{\mathrm{x}}=2.0 \mathrm{~s}^{\prime} \quad \omega_{\mathrm{y}}=2.0 \mathrm{~s}^{-1} \quad \omega_{\mathrm{z}}=2.0 \mathrm{~s}$

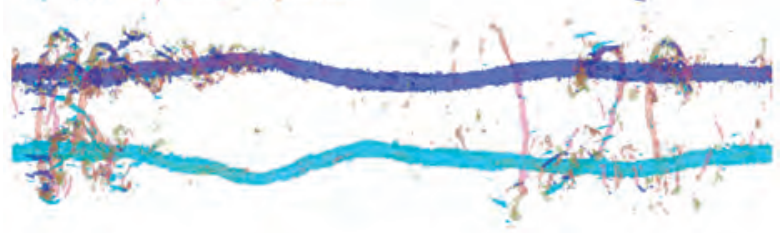

(h)

Time $=83.85 \mathrm{sec}$

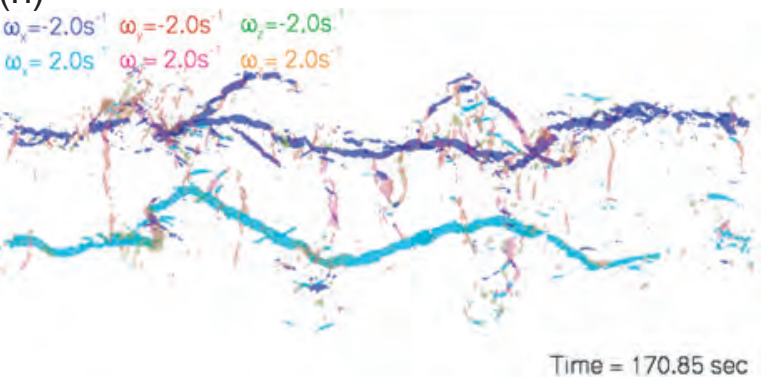

Figure 10. Time evolution of wake shown by iso-surface of vorticity magnitude, where positive and negative values from each vorticity component is used. 


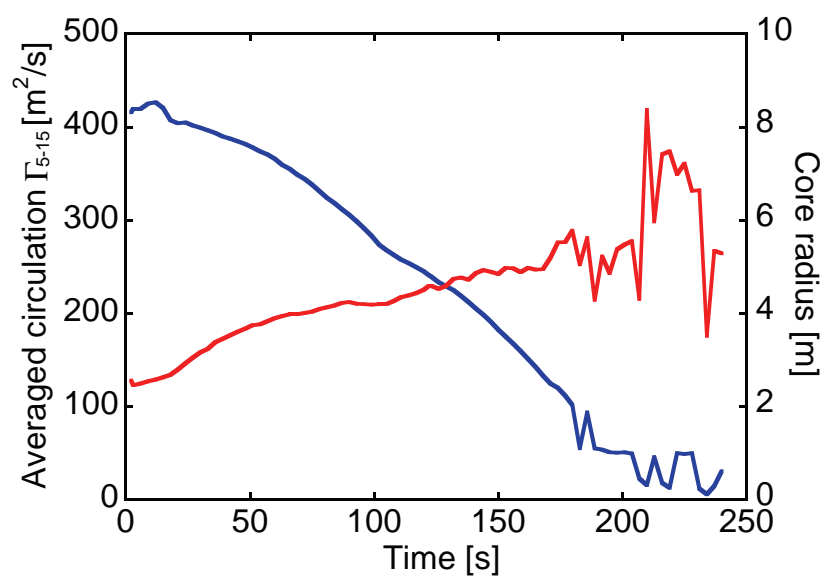

Figure 11. Time evolution of averaged circulation and vortex core radius.

\section{Conclusions}

LES of wake vortex evolution from vortex generation to decay is performed by combining solutions from RANS and LES computations. Velocity distribution around the DLR-F6 model obtained from RANS simulation is used to initialize wake vortex by sweeping it through a ground-fixed LES domain. Steady RANS solution is employed in LES computation as a constant forcing term during the initialization of wake. The transition between RANS and LES is realized by a hyperbolic tangent function where wall-distance from the model surface is used as a threshold.

Parameters for the integration procedure of RANS and LES as well as mesh resolution in LES have been investigated. Finer mesh resolution realizes higher peaks of vorticity distribution in the wake. However, the global structure of aircraft wake is already achieved with the coarse mesh resolution. It turns out that coarser mesh resolution, i.e., lower vorticity peak results in lower circulation than the value calculated from aerodynamic lift. A parametric study of the weighting function parameters indicates that smoother transition and larger overlap region realize smoother vorticity distribution in the aircraft wake. Currently, relatively large threshold values of 4.0 to $6.0 \mathrm{~m}$ are used, however, finer mesh resolution in LES would make these smaller to realize similar results.

Based on the wake initialized by the DLR-F6 model, LES is performed until the time of vortex decay. Vorticity production from the fuselage was relatively large in the present case, however, it decays quickly unlike the wing-tip vortex. Vorticity from inboard wing is also relatively large, which has same rotation orientation with the wing-tip vortex and it is merged during time evolution. Periodic boundary conditions currently used in this phase induce disturbances from the boundary, which result in earlier decay of the wake vortex.

For future work, the influence of mesh resolution needs to be further investigated. Detailed flow structures and vorticity peaks should be captured to obtain a reasonable circulation value of a rolled-up vortex pair. Another issue is a periodic boundary condition after wake initialization. The time evolution of wake vortex is sensitive to physical and numerical disturbances, therefore, the numerical disturbances originated from a boundary condition need to be carefully reduced to investigate the effect of physical disturbances. In addition, turbulence fluctuation modeled as eddy viscosity in RANS simulation could be considered explicitly in LES.

\section{Acknowledgments}

The provision of the RANS solution of the DLR-F6 model by Olaf Brodersen, Niko Schade and Bernhard Eisfeld of Institut für Aerodynamik und Strömungstechnik, DLR, Braunschweig is greatly acknowledged. The supply of computation time on the supercomputers IBM p575 system at Deutsches Klimarechenzentrum (DKRZ) and SGI Altix 4700 system at Leibniz-Rechenzentrum (LRZ) is also greatly acknowledged. The current work has been conducted within the DLR project Wetter \& Fliegen (Weather \& Flying). 


\section{References}

${ }^{1}$ Gerz, T., Holzäpfel, F., and Darracq, D., "Commercial Aircraft Wake Vortices," Progress in Aerospace Science, Vol. 38, No. 3, 2002, pp. 181-208.

${ }^{2}$ Minnis, P., Young, D. F., Ngyuen, L., Garber, D. P., Jr., W. L. S., and Palikonda, R., "Transformation of contrails into cirrus during SUCCESS," Geophysical Research Letters, Vol. 25, No. 8, 1998, pp. 1157-1160.

${ }^{3}$ van Dam, C. P., "The aerodynamics design of multi-element highlift systems for transport airplanes," Progress in Aerospace Science, Vol. 38, No. 3, 2002, pp. 101-144.

${ }^{4}$ Gerz, T., Dürbeck, T., and Konopka, P., "Transport and Effective Diffusion of Aircraft Emissions," Journal of Geophysical Research, Vol. 103, No. D20, 1998, pp. 25,905-25,913, Passive tracer simulation.

${ }^{5}$ Stumpf, E., "Study of Four-Vortex Aircraft Wakes and Layout of Corresponding Aircraft Configurations," Journal of Aircraft, Vol. 42, No. 3, 2005, pp. 722-730.

${ }^{6}$ Leweke, T. and Williamson, C. H. K., "Cooperative Elliptic Instability of a Vortex Pair," Journal of Fluid Mechanics, Vol. 360 .

${ }^{7}$ Nomura, K. K., Tsutsui, H., Mahoney, D., and Rottman, J. W., "Short-Wavelength Instability and Decay of a Vortex Pair in a Stratified Fluid," Journal of Fluid Mechanics, Vol. 553.

${ }^{8}$ Laporte, F. and Corjon, A., "Direct Numerical Simulation of the Elliptic Instability of a Vortex Pair," Physics of Fluids, Vol. 12, No. 5, 2000, pp. 1016-1031.

${ }^{9}$ Han, J., Lin, Y., Schowalter, D. G., and Pal Arya, S., "Large Eddy Simulation of Aircraft Wake Vortices Within Homogeneous Turbulence: Crow Instability," AIAA Journal, Vol. 38, No. 2, 2000, pp. 292-300.

${ }^{10}$ Proctor, F. H., Hamilton, D. W., and Han, J., "Wake Vortex Transport and Decay in Ground Effect: Vortex Linking with the Ground," AIAA Paper 2000-757.

${ }^{11}$ Holzäpfel, F., Gerz, T., Frech, M., and Dornbrack, A., "Wake Vortices in Convective Boundary Layer and Their Influence on Following Aircraft," Journal of Aircraft, Vol. 37, No. 6, 2000, pp. 1001-1007.

${ }^{12}$ Holzäpfel, F., Gerz, T., and Baumann, R., "The Turbulent Decay of Trailing Vortex Pairs in Stably Stratified Environments," Aerospace Science and Technology, Vol. 5, No. 2, 2001, pp. 95-108.

${ }^{13}$ Holzäpfel, F., Misaka, T., and Hennemann, I., "Wake-Vortex Topology, Circulation, and Turbulent Exchange Processes," AIAA Paper 2010-7992.

${ }^{14}$ Paugam, R., Paoli, R., and Cariolle, D., "Influence of Vortex Dynamics and Atmospheric Turbulence on the Early Evolution of a Contrail," Atmospheric Chemistry and Physics, Vol. 10, 2010, pp. 3922-3952.

${ }^{15}$ Fujii, K., "Unified Zonal Method Based on the Fortified Solution Algorithm," Journal of Computational Physics, Vol. 118, No. 1, 1995, pp. 92-108.

${ }^{16}$ Kalnay, E., Atmospheric Modeling, Data Assimilation and Predictability, Cambridge University Press, 2003.

${ }^{17}$ Hennemann, I. and Holzäpfel, F., "Aircraft Wake Vortex Curvature and Resulting Risk Potential for Following Aircraft," CEAS 2009 European Air and Space Conference, Munchester, UK.

${ }^{18}$ Manhart, M., "A Zonal Grid Algorithm for DNS of Turbulent Boundary Layer," Computer and Fluids, Vol. 33, No. 3, 2004, pp. 435-461.

${ }^{19}$ Gerz, T. and Ehret, T., "Wingtip Vortices and Exhaust Jets During the Jet Regime of Aircraft Wakes," Aerospace Science and Technology, Vol. 1, No. 7, 1997, pp. 463-474.

${ }^{20}$ Bannon, P. R., "Potential Vorticity Conservation, Hydrostatic Adjustment, and the Anelastic Approximation," Journal of the Atmospheric Sciences, Vol. 52, No. 12, 1995, pp. 2302-2312.

${ }^{21}$ Kobayashi, M. H., "On a Class of Páde Finite Volume Methods," Journal of Computational Physics, Vol. 156, No. 1, 1999, pp. 137-180.

${ }^{22}$ Hokpunna, A. and Manhart, M., "Compact Fourth-order Finite Volume Method for Numarical Solutions of navier-Stokes Equations on Staggered Grids," Journal of Computational Physics, Vol. 229, No. 20, 2010, pp. 7545-7570.

${ }^{23}$ Hokpunna, A., "Compact Fourth-order Scheme for Numerical Simulations of Navier-Stokes Equations," Ph.D Thesis, Technische Universität München, Germany.

${ }^{24}$ Hirt, C. W. and Cook, J. L., "Calculating Three-dimensional Flows Around Structures and Over Rough Terrain," Journal of Computational Physics, Vol. 10, No. 2, 1972, pp. 324-340.

${ }^{25}$ Williamson, J. H., "Low-storage Runge-Kutta Schemes," Journal of Computational Physics, Vol. 35, No. 48, 1980, pp. $48-56$.

${ }^{26}$ Meneveau, C., Lund, T. S., and Cabot, W. H., "A Lagrangian Dynamic Subgrid-scale Model of Turbulence," Journal of Fluid Mechanics, Vol. 319.

${ }^{27}$ Shen, S., Ding, F., Han, J., Lin, Y., Arya, S. P., and Proctor, F. H., "Numerical Modeling Studies of Wake Vortices: Real Case Simulations," AIAA Paper 99-0755.

${ }^{28}$ Holzäpfel, F., "Adjustment of Subgrid-Scale Parameterizations to Strong Streamline Curvature," AIAA Journal, Vol. 42, No. 7, 2004, pp. 1369-1377.

${ }^{29}$ Brodersen, O., Eisfeld, B., Raddatz, J., and Frohnapfel, P., "DLR Results from the Third AIAA Computational Fluid Dynamics Drag Prediction Workshop," Journal of Aircraft, Vol. 45, No. 3, 2008, pp. 823-836.

${ }^{30}$ Schlüter, J. U., Wu, X., Kim, S., Shankaran, S., Alonso, J. J., and Pitsch, H., "A Framework for Coupling ReynoldsAveraged With Large-Eddy Simulations for Gas Turbine Applications," Transaction of ASME, Journal of Fuilds Engineering, Vol. 127, 2005, pp. 806-815.

${ }^{31}$ Crow, S. C., "Stability Theory for a Pair of Trailing Vortices," AIAA Journal, Vol. 8, No. 12, 1970, pp. 2172-2179. 